POS PROCEEDINGS

\title{
Blue Early Type Galaxies with the MeerKAT
}

\section{G. I. G. Józsa*}

SKA South Africa, The Park, Park Road, Pinelands, 7405, South Africa

Rhodes University, RARG, RATT, PO Box 94, Grahamstown, 6140, South Africa

Argelander-Institut für Astronomie, Auf dem Hügel 71, D-53121 Bonn, Germany

E-mail: jozsa@ska.ac.za

\section{O. Ivy Wong}

International Centre for Radio Astronomy Research, The University of Western Australia M468, 35 Stirling Highway, Crawley, WA 6009, Australia

E-mail: ivy.wongeuwa.edu.au

\section{Thomas Mauch}

SKA South Africa, The Park, Park Road, Pinelands, 7405, South Africa

E-mail: tmaucheska.ac.za

\section{Kevin Schawinski}

Institute for Astronomy, ETH Zürich, Wolfgang-Pauli-Strasse 27, 8093 Zürich, Switzerland.

E-mail: kevin.schawinski@phys.ethz.ch

\section{Chandreyee Sengupta}

Korea Astronomy and Space Science Institute, 776, Daedeokdae-ro, Yuseong-gu, Daejeon 305-348, Korea

E-mail: sengupta.chandreyee@gmail.com

\section{Karen Masters}

Institute of Cosmology Gravitation, University of Portsmouth, Dennis Sciama Building,

Portsmouth, PO1 3FX, UK

South East Physics Network (SEPNet)

E-mail: karen.masters@port.ac.uk

\section{Meg Urry}

Yale Center for Astronomy and Astrophysics and Department of Physics, Yale University, P.O. Box 208120, New Haven, CT 06520-8120, USA

E-mail: meg.urry@yale.edu

\section{Chris Lintott}

Oxford Astrophysics, Denys Wilkinson Building, Keble Road, Oxford OX1 3RH, UK

E-mail: cjleastro.ox.ac.uk

\section{Brooke Simmons}

University of California San Diego, CASS 0424, 9500 Gilman Drive, La Jolla, CA 92093, USA

E-mail: bdsimmonseucsd. edu

\section{Sugata Kaviraj}


Oxford Astrophysics, Denys Wilkinson Building, Keble Road, Oxford OX1 3RH, UK

Centre for Astrophysics Research, University of Hertford-shire, College Lane, Hatfield, Herts,

AL10 9AB, UK

E-mail: s.kavirajeherts.ac.uk

\section{Peter Kamphuis}

National Centre for Radio Astrophysics, TIFR, Ganeshkhind, Pune 411007, India

E-mail: peterkamphuisastronomyegmail.com

We discuss a potential MeerKAT campaign to shed more light into the nature of the optically identified Blue Early Type galaxies (BETGs), a subset of the so-called "green valley" population, quenching star formation on time scales of less than $0.25 \mathrm{Gyr}$ and the likely progenitor population of post-starburst galaxies. Employing a WSRT pilot survey of four galaxies, we have shown that BETGs have radio properties that fit to a mechanical removal of star forming material, potentially by AGN activity, as in particular we found $\mathrm{H}$ I to be removed farther and farther from the centre of the galaxies with older and older age of the stellar population. We argue that MeerKAT is the optimal instrument to conduct a pointed survey of BETGs to become more conclusive about this specific transition state, which is so rare that it will not be detected at a high enough rate in planned sufficiently sensitive large-sky surveys, for which we expect a total detection number of the order of 12 objects.

MeerKAT Science: On the Pathway to the SKA,

25-27 May, 2016,

Stellenbosch, South Africa

${ }^{*}$ Speaker. 


\section{Blue Early Type Galaxies}

It is well known that galaxies can roughly be divided into two morphological categories, into elliptical or early-type galaxies and late-type or spiral galaxies [1], the first being characterised as having an ellipsoid shape and being gas-poor and forming little to no stars, the latter being flattened disks and in general gas-rich and actively forming stars.

Large sky surveys such as the Sloan Digital Sky Survey (SDSS) [2] were used to quantify a strong colour bimodality of galaxies, which has been roughly identified to be strongly correlated with the morphological bimodality [3]. Most galaxies are either blue and star-forming galaxies or they are red and passively-evolving. This bimodality is already evident in simple histograms [3], but becomes most evident in a scatter diagram showing the colour of galaxies vs. their absolute magnitude[4]. Galaxies mostly reside within the "blue cloud" or are red passively-evolving galaxies that reside in the "red sequence" in the colour-magnitude diagram, a tendency that is even enhanced when replacing absolute magnitude by stellar mass [5]. The absence of a significant galaxy population in the intervening region (often referred to as the "green valley") between these two populations infers that the transition between the two main galaxy populations must occur relatively quickly [6], making the green valley a specifically interesting region in the colour-magnitude diagram.

While the colour-magnitude diagram hence largely reveals two populations that can be distinguished by their colour, no sharp division of the two types occurs in the colour-magnitude diagram. It contains spiral galaxies which are redder than their average morphological type[7] and passively evolving, as well as early type galaxies which are particularly blue, and star forming, the BETGs [8]. Actively forming stars or at least having had a very recent period of active star fomation, they are thought to be potential progenitors of post-starburst E+A galaxies [8,9].

An even more detailed look making use of an optical identification of the galaxy type, reveals that the transition region with mixed morphological types in the colour-magnitude diagram is larger than naively expected [10]. In other words the colour bimodality does not perfectly reflect the morphological one and it is instructive to investigate the colour-magnitude diagrams of both morphological types separately. Schawinski et al. [10] show that indeed while early type galaxies with a blue colour exist, they are rare, and populate the green valley only sparsely. Disk galaxies, on the contrary, broadly populate the green valley and exist with a large variety in colour and, with that, star formation properties, without any significant evidence of a suppression in the green valley. This suggests that BETGs are fast evolving galaxies, while red disk galaxies are slowly, passively evolving galaxies. These findings are supported by the analysis of colour-colour diagrams using UV- and optical colours. On average, late-type galaxies exhibit a light profile indicative of a higher proportion of very young stars in comparison to an intermediate population, meaning that star formation is shut down more rapidly in early-type galaxies populating the green valley than in late-type galaxies. Making use of evolutionary models, Schawinski et al. [10] argue that on average red late-type galaxies evolve passively, exhausting their star formation reservoir, with a quenching time of $\tau_{\text {quench }}>2 \mathrm{Gyr}$, while BETGs have a much shorter quenching time $\left(\tau_{\text {quench }}<0.25 \mathrm{Gyr}\right.$ ), indicating a mechanical removal of the star forming material from the galaxies. Showing that at the same time late-type galaxies become redder with increasing mass, they propose that quenching star formation in late-type galaxies is mostly due to a gradual reduction of accretion, while for late-type 
galaxies a more instantaneous, mechanical, gas removal is required to explain the observations and propose explicitly feedback by active galactic nuclei as a potential quenching mechanism.

Our recent stellar population study of early- and late-type galaxies find that there are two main modes of quenching star formation in galaxies $-\mathrm{a}$ fast $\left(\tau_{\text {quench }}<0.25 \mathrm{Gyr}\right)$ mode in early-type galaxies (ETGs) and a slow ( $\tau_{\text {quench }}>2$ Gyr) mode in late-type galaxies (LTGs) [10].

\section{A radio campaign to characterise Blue Early Type Galaxies}

We are pursuing a radio campaign to characterise BETGS in the radio regime, in particular focusing on the $\mathrm{H}$ I line and L-band continuum. The purpose of this project is to get better insight in the physical mechanism(s) that cause(s) the rapid shutdown of star formation in galaxies and/or to determine whether BETGs are even more complex than laid out above and more pathways may lead to the same evolutionary stage, like e.g. a (re-)accretion period of star forming material, initiating star formation in a former passively evolving galaxy [11, 12, 13, 14, 15].

Previous studies on this topic have been largely focussed on post-starburst galaxies (PSGs), galaxies which are no longer forming stars but still have a dominant population of recently-formed stars $[16,17,18,19,20,21,22,23,9]$. Our recent study of low redshift $(0.02<z<0.05)$ PSGs found that these galaxies typically have spheroidal early-type morphologies [9] and are already too evolved to still exhibit the signatures for star formation quenching [9, 24]. Therefore, we posit that the smoking gun for rapid star formation quenching is more likely to be found in its proposed predecessor population, namely, the BETGs [24].

As laid out above, BETGs do not fit the canonical bimodal scheme of red ellipticals versus blue spirals. They are unlikely descendants of blue spirals [25]. These blue ETGs are likely to be transition-type galaxies and are likely predecessors of low redshift PSGs. A sample of 204 low redshift $(0.02<z<0.05)$ blue ETGs are identified using SDSS and the Galaxy Zoo project [8]. The blue ETGs account for 5.7\% of all ETGs found within the same redshift range and are very rare in the Local Universe [8]. Previous CO observations of blue ETGs show that the molecular gas reservoirs are being rapidly destroyed during the composite star formation and AGN phase [26].

The neutral gas content (as traced by H I) is an excellent indicator of the potential for future star formation as well as an excellent tracer of past interactions. Evidence of past interactions in a galaxy can be found imprinted in its $\mathrm{H}$ I gas morphology and kinematics because the $\mathrm{H}$ I reservoir typically extends to larger radii than the stellar component within a galaxy and is more susceptible to the tidal torques incurred by interactions. In addition, $\mathrm{H}$ I observations also provide simultaneous observations of $1.4 \mathrm{GHz}$ radio continuum emission which is an excellent tracer of activity from the galaxy's Active Galactic Nuclei (AGN).

\subsection{A pilot survey with the WSRT}

Starting our campaign, we completed a pilot H I survey of four Northern BETGs using the Westerbork Synthesis Radio Telescope (WSRT)[24]. These four pilot galaxies are selected to represent 4 separate stages spanning the entire range of post-quenching evolution. The purpose of this pilot survey is to help prepare for our large observational campaign to map the $\mathrm{H}$ I gas in the nearest BETGs. Subsequently, we have recently conducted HI observations with the GMRT and 


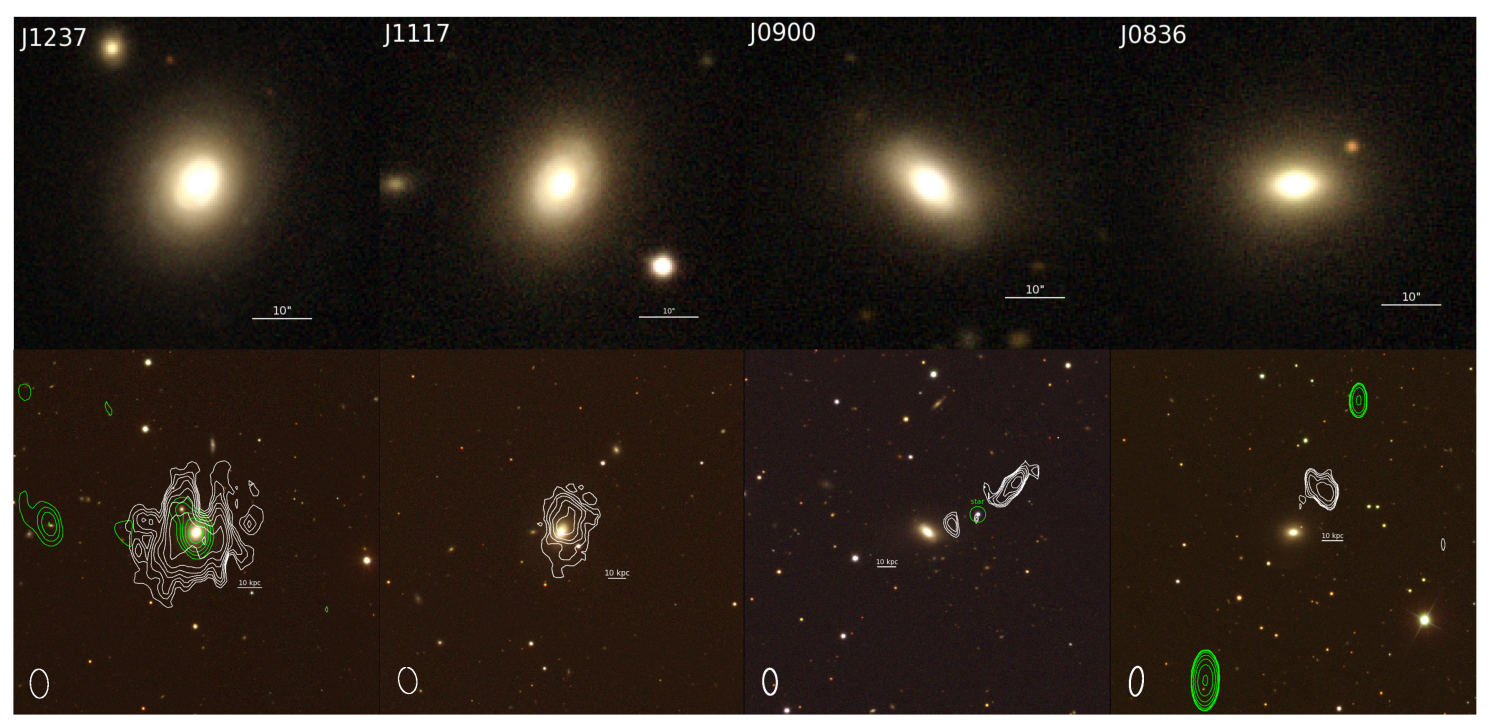

Figure 1: Top row: optical gri SDSS composite images of the four blue early-type galaxies in our sample. The galaxies are arranged from left to right in terms of $N U V-u$ colour from blue to red as a tracer of time. The white scale bars represent 10 arcseconds. Bottom row: zoomed-out optical images with the WSRT radio observations overlaid. The white contours (asinh-stretch) represent the H I and the green contours (logarithmic stretch) the radio continuum. The lowest radio and $\mathrm{H}$ I contours begin at $3 \sigma$ and the highest contour is matched to the peak flux density of $0.87 \mathrm{mJy}$ beam $^{-1}, 1.51$ mJy beam $^{-1}, 0.86 \mathrm{mJy}_{\text {beam }}{ }^{-1}, 44.79 \mathrm{mJy}_{\text {beam }}{ }^{-1}$ for J1237, J1117, J0900 and J0836; respectively. It should be noted that the nuclear $1.4 \mathrm{GHz}$ continuum point sources are not shown for J0900 and J1117 to avoid confusion with the H I contours. [24]

the VLA and have managed to increase our sample by two galaxies. We are currently in the process of analysing these extra 2 galaxies.

From the pilot survey, we find increasing projected spatial offsets between the main $\mathrm{H}$ I reservoir and the stellar component of the galaxies with advancing quenching age, $\tau_{\text {quench }}[24]$. In the least-evolved case (J1237), the $\mathrm{H}$ I content is comparable to other star-forming galaxies with similar $N U V-r$ colours and is observed to be undisturbed and in stable rotation. However, as $\tau_{\text {quench }}$ increases, the gas-to-stellar fraction decreases and the $\mathrm{H}$ I reservoir begins to exhibit increasingly disturbed morphologies and kinematics. In the case of the most evolved pilot galaxy (J0836), we also observe a pair of $1.4 \mathrm{GHz}$ radio lobes flanking both sides of the galaxy and aligned with the displaced H I reservoir [24]. The presence of these radio lobes suggest past AGN nuclear activity. Due to the alignment between the observed radio lobes and the H I cloud, as well as the lack of a neighbouring galaxy with which J0836 could have interacted; we hypothesise that the H I reservoir could have been pushed out by a previous episode of AGN activity associated with the observed radio lobes. Figure 1 shows the $\mathrm{H}$ I and radio continuum observations overlaid as white and green contours, respectively, on the optical SDSS observations of the four pilot galaxies.

The gas fractions of blue ETGs (Fig. 2) are generally lower compared to the ones of gasrich star-forming galaxies from the ALFALFA H I survey [27] — consistent with the idea that the process of quenching has already begun in these galaxies. Relative to the results from the Atlas3D survey [28] of nearby ETGs, we find that the Atlas3D ETGs span a large range of gas fractions for a given $N U V-r$ colour. Of particular note is that every Atlas3D ETG exhibiting a disturbed 


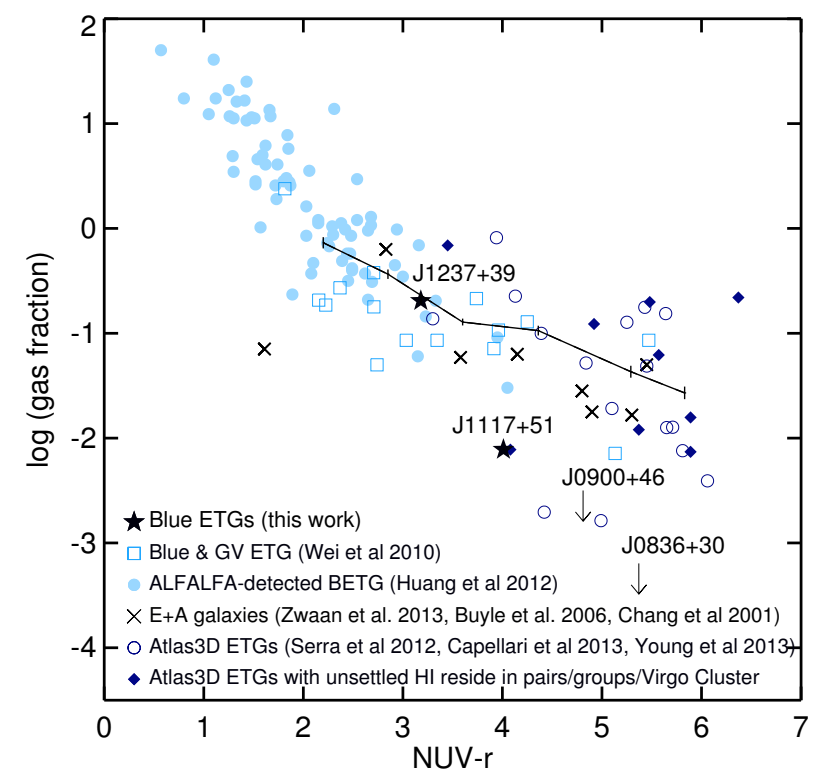

Figure 2: H I-to-stellar mass ratio as a function of galaxy colour. The solid line shows the average gas fractions found from GASS survey of massive transition-type galaxies [29].

or unsettled H I morphology reside in pairs or groups or the Virgo Cluster, and have higher gas fractions than what we find in our pilot sample of isolated blue ETGs. Similarly, the massive transition-type galaxies studied by the GASS survey [29] also has higher average gas fractions. The same is true for a sample of 14 blue-sequence E/S0s analysed by Wei et al. 2010 [12], argued to be in a likely status of reaccretion. This sample would likely have fallen out of our pilot sample as also the morphology of the galaxies is indicative of being non-elliptical and/or interacting, consistent with their gas fraction.

We find our gas fractions to be consistent with the idea that our pilot sample have shorter $\tau_{\text {quench }}$ than those investigated by these previous studies.

Our pilot study is hence consistent with the hypothesis that the fast star formation shutdown in blue ETGs is due to the bulk removal of neutral gas from which stars can form (possibly by an active AGN exhibiting radio jets or outflows) rather than the slow exhaustion of gas via star formation. In particular, if the opposite path would be true, for our four galaxies, it would be expected that the $\mathrm{H}$ I gas reservoir is likely larger for galaxies with lower recent star formation, as well as the absence of an intermediate stellar population (as traced by the blue light) in galaxies with little ongoing or very recent star formation (see Fig. 2). Clearly, the sample is not large enough to be conclusive, which is why we continue our campaign. There are open questions than can only be answered with a more statistically-representative sample of galaxies. How are interactions and AGN related in these blue ETGs as they evolve into PSGs and subsequently, passive ETGs? Does AGN feedback result in $\mathrm{H}$ I outflows? Are the displaced $\mathrm{H}$ I reservoirs a result of previous mergers? How many BETGs may be a result of gas capture instead of quenching? 


\subsection{A MeerKAT campaign to observe Blue Early Type Galaxies}

As laid out above, MeerKAT with its predicted sensitivity is the most suitable instrument to conduct our proposed survey. Our aim would be a comparably small campaign with a potentially high impact. Our intended sample size is 35 BETGs in total We estimate an integration time of 2 hours per pointing with the full array to be sufficient to detect a column density of $5 \cdot 10^{19}$ atoms $\mathrm{cm}^{-2}$ over a line width of $20 \mathrm{~km} \mathrm{~s}^{-1}$. This column density limit is at least required to detect low-column density gas in these quenched objects at the given resolution, which itself is required to resolve the $\mathrm{H}$ I to determine its position and status as shown in our pilot survey. The intention would be to begin with observations of the nearest BETGs using early MeerKAT-32 (or earlier) from a sample of Southern BETGs as identified by Schawinski et al. (2009) [8], with the integration time scaled up accordingly (8 hours per target). Observations from SkyMapper help us to determine a more complete sample of Southern BETG. We expect to find several hundred Southern BETG in addition to the sample reported by Schawinski et al 2009 to select our sub-sample. We hence intend to define a small survey to start with MeerKAT-32 to then continue with MeerKAT-64, for which the total integration time (without calibration) would be 70 hours. We emphasise that, given the small local density of BETGs, none of the planned blind H I surveys will yield serendipitous detections of a sufficient number of BETGs at sufficient resolution. Schawinski et al. (2009) [8] find 204 BETGs within the survey area of SDSS (DR 6, 9600 deg ${ }^{2}$ ) within a redshift range of 0.2-0.5. With an aspired H I 5- $\sigma_{\text {rms }}$ detection limit of $2 \cdot 10^{8} M_{\odot}$ we extrapolate this to 3 BETGs that can be detected in the MIGHTEE survey, 8 to 9 in the APERTIF medium-deep survey, while shallower surveys (APERTIF shallow and WALLABY) would not provide the required resolution $\left(15^{\prime \prime}\right.$ at the targeted column-density sensitivity). Only MeerKLASS (see contribution of M. Santos in this conference) would provide a comparable sample.

\section{Summary}

We discuss an H I survey of Blue Early Type Galaxies as a potential small campaign with the MeerKAT. We argue that MeerKAT is an optimal telescope to conduct a galaxy survey of a rare transition type as it has a very sensitive single-pixel receiver. We discuss the characteristics of BETGs as defined by Schawinski et al. (2009) [8] and argue that they are most likely the result of a fast star formation quenching event, potentially AGN feedback. We use pilot WSRT observations to support this hypothesis [24] and argue that a larger survey of this type is likely to shed more light into the nature of BETGs. A survey of this kind is not extremely expensive and a very good example for a high-yield special-purpose survey.

\section{References}

[1] Hubble, E. P. 1926, ApJ, 64, 321

[2] York, D.G., et al. 2000, AJ, 120, 1579

[3] Strateva, I., et al. 2001, AJ, 122, 1861

[4] Baldry I. K., Glazebrook K., Brinkmann J., Ivezic Z., Lupton R. H., Nichol R. C., Szalay A. S., 2004, ApJ, 600, 681 
[5] Baldry, I. K., Balogh, M. L., Bower, R. G., Glazebrook, K., Nichol, R. C., Bamford, S. P., Budavari, T. 2006, MNRAS, 373, 469

[6] Martin, D.C., et al. 2007, ApJS, 173, 415

[7] Masters, K. L., et al. 2010, MNRAS, 405, 783

[8] Schawinski K. et al., 2009, MNRAS, 396, 818

[9] Wong, O. I., et al. 2012, MNRAS, 420,1684

[10] Schawinski, K., et al. 2014, MNRAS, 440, 889

[11] Kannappan, S. J., Guie, J. M., Baker, A. J. 2009, AJ, 138, 579

[12] Wei, L. H., Kannappan, S. J., Vogel, S. N., Baker, A. J. 2010, ApJ, 708, 841

[13] Wei, L. H., Vogel, S. N., Kannappan, S. J., Baker, A. J., Stark, D. V., Laine, S. 2010, ApJ, 725, L62

[14] Moffett, A. J., Kannappan, S. J., Baker, A. J., Laine, S. 2012, ApJ, 745, 34

[15] Stark, D. V., Kannappan, S. J., Wei, L. H., Baker, A, J., Leroy, A. K., Eckert, K. D., Vogel, S. N. 2013, ApJ, 769, 82

[16] Dressler, A., Gunn, J. E. 1983, ApJ, 270, 7

[17] Couch, W. J., Sharples, R. M. 1987, MNRAS, 229, 423

[18] Poggianti, B.M., et al. 1999, ApJ, 518, 576

[19] Tran, K., et al. 2004, ApJ, 609, 683

[20] Goto, T. 2005, MNRAS, 357, 937

[21] Yan, R., et al. 2009, MNRAS, 398, 735

[22] Wild, V., et al. 2008, MNRAS, 395, 144

[23] Zwaan, M.A., et al. 2013, MNRAS, 432, 492

[24] Wong, O. I., et al. 2015, MNRAS, 447, 3311

[25] Tojeiro, R., et al. 2013, MNRAS, 432, 359

[26] Schawinski, K., et al. 2009, MNRAS, 690, 1672

[27] Huang, S., et al. 2012, ApJ, 756, 113

[28] Serra, P., et al. 2012, MNRAS, 422, 1835

[29] Catinella, B., et al. 2012, A\&A, 544, A65 